\title{
Effects of Prior Data on the Inference and Filtering Based Electrocardiographic Imaging
}

\author{
Taha Erenler ${ }^{1}$, Yesim Serinagaoglu Dogrusoz ${ }^{1}$ \\ ${ }^{1}$ Electrical and Electronics Engineering Department, Middle East Technical University, Turkey
}

\begin{abstract}
Statistical estimation techniques with "good" prior information improves the accuracy of electrocardiographic imaging (ECGI). Obtaining a "good" prior information in terms of training data, and how to use these data to estimate the prior parameters, are among of the primary challenges in statistical ECGI literature. This study investigates the effects of training set compositions, and prior parameter estimation methods on the ECGI accuracy. Two different training sets were used to determine the prior statistical parameters: 1) Beats that are all paced close to the test beat pacing site, 2) Beats with pacing sites covering a wider region around the test beat pacing site. These two training sets are obtained from a database of previously recorded epicardial potentials and used in maximum likelihood (ML)- and maximum a posteriori (MAP)-based prior estimation methods. The inverse problem is then solved by using the Kalman filter, based on those priors. Our results show that the Tikhonov regularization is the most fragile method to the measurement noise. MAPIF method is more robust to measurement noise in terms of electrogram reconstruction and activation time estimation accuracies, for both training sets. MLIF performed better with a more spread training set in terms of electrogram reconstruction accuracy, but showed better activation time reconstruction performance with the first training set.
\end{abstract}

\section{Introduction}

In ECGI, one can enhance the accuracy of inverse solutions by including precise prior information. For example, Serinagaoglu et al. [1] investigated the effects of prior information on the solution. They improved the accuracy of solutions by including epicardial potential measurements which were taken directly from the subject's heart in addition to epicardial potentials which were taken from a database. Furthermore, in deterministic methods' context, Gutierrez et al. [2] also studied the importance of prior information and developed a new method which modifies zero order Tikhonov regularization by taking into account the measurements near endocardium region. Generally, including a priori information yields more accurate solutions in these two studies. Recently, in [3], we presented a study on ECGI by using ML and MAP estimation methods. In that study, the training sets were selected by following a more general manner, meaning that no systematic way was followed to choose the training sets. To understand the effects of prior information on ML and MAP methods more profoundly, in this study, we follow a systematic approach to choose the training sets and investigate the impacts of these prior information on the solution.

\section{State-Space Formulation of the Prob- lem}

Here, the epicardial potentials are used as cardiac sources. Hence, the problem can be formulated in a linear state-space form as follows:

$$
\begin{aligned}
\mathbf{y}_{k} & =\mathbf{A} \mathbf{x}_{k}+\mathbf{v}_{k}, \\
\mathbf{x}_{k+1} & =\mathbf{F} \mathbf{x}_{k}+\mathbf{w}_{k}
\end{aligned}
$$

where $\mathbf{x}_{k} \in \mathbb{R}^{\mathbb{N}}$ and $\mathbf{y}_{k} \in \mathbb{R}^{\mathbb{M}}$ denote the epicardial and the body surface potential vectors at time $k$, respectively; $\mathbf{v}_{k} \in \mathbb{R}^{\mathbb{M}} \sim \mathcal{N}\left(\mathbf{v}_{k} ; \mathbf{0}, \mathbf{R}\right)$ and $\mathbf{w}_{k} \in \mathbb{R}^{\mathbb{N}} \sim \mathcal{N}\left(\mathbf{w}_{k} ; \mathbf{0}, \mathbf{Q}\right)$ correspond to measurement and process noises, respectively; $\mathbf{F}$ is the $N \times N$ state transition matrix and $\mathbf{A}$ is the $M \times N$ forward transfer matrix. Furthermore, the process and measurement noises are assumed to be uncorrelated with the state $\mathbf{x}_{k}$.

\section{Kalman Filtering and Smoothing}

Kalman filter is an optimal estimator used for reconstructing the unobserved states of a system by using the observed data. There are two stages in the Kalman filter. In the first stage, the predicted state vector $\mathbf{x}_{k \mid k-1}$ and the state covariance are estimated as:

$$
\begin{aligned}
\mathbf{x}_{k \mid k-1} & =\mathbf{F x}_{k-1 \mid k-1} \\
\mathbf{P}_{k \mid k-1} & =\mathbf{F P}_{k-1 \mid k-1} \mathbf{F}^{T}+\mathbf{Q}
\end{aligned}
$$


Second stage is about updating the predictions $\mathbf{x}_{k \mid k-1}$ and $\mathbf{P}_{k \mid k-1}$ by using the measurement vector $\mathbf{y}_{k}$ to estimate the state vector, $\mathbf{x}_{k \mid k}$, and the state covariance matrix, $\mathbf{P}_{k \mid k}$ as follows:

$$
\begin{aligned}
\mathbf{K}_{k} & =\mathbf{P}_{k \mid k-1} \mathbf{A}^{T}\left(\mathbf{A} \mathbf{P}_{k \mid k-1} \mathbf{A}^{T}+\mathbf{R}\right)^{-1} \\
\mathbf{x}_{k \mid k} & =\mathbf{x}_{k \mid k-1}+\mathbf{K}_{k}\left(\mathbf{y}_{k}-\mathbf{A} \mathbf{x}_{k \mid k-1}\right) \\
\mathbf{P}_{k \mid k} & =\left(\mathbf{I}-\mathbf{K}_{k} \mathbf{A}\right) \mathbf{P}_{k \mid k-1}
\end{aligned}
$$

where $\mathbf{K}_{k}$ is the Kalman gain. In order to initialize the Kalman filter at the first time instant and run the filter recursively, the mean vector $\overline{\mathbf{x}}_{1}=\mathbf{x}_{0 \mid 0}$ and covariance matrix $\boldsymbol{\Sigma}_{1}=\mathbf{P}_{0 \mid 0}$, must be found. Furthermore, the state transition matrix $\mathbf{F}$, and the error covariances $\mathbf{R}$ and $\mathbf{Q}$ also must be specified.

After the Kalman filter results are found, Rauch-TungStriebel Smoother (RTS) [4] is used to perform a second set of recursions from the last time instant down to the first time instant according to the following equations:

$$
\begin{aligned}
\mathbf{P}_{k+1 \mid k} & =\mathbf{F} \mathbf{P}_{k \mid k} \mathbf{F}^{T}+\mathbf{Q} \\
\mathbf{G}_{k} & =\mathbf{P}_{k \mid k} \mathbf{F}^{T}\left(\mathbf{P}_{k+1 \mid k}\right)^{-1} \\
\mathbf{x}_{k \mid T}^{s} & =\mathbf{x}_{k \mid k}+\mathbf{G}_{k}\left[\mathbf{x}_{k+1 \mid T}-\mathbf{F} \mathbf{x}_{k \mid k}\right] \\
\mathbf{P}_{k \mid T}^{s} & =\mathbf{P}_{k \mid k}+\mathbf{G}_{k}\left(\mathbf{P}_{k+1 \mid T}^{s}-\mathbf{P}_{k+1 \mid k}\right) \mathbf{G}_{k}^{T}
\end{aligned}
$$

The inverse solution at the $k^{\text {th }}$ time instant for the Kalman filter and smoother are $\mathbf{x}_{k \mid k}$ and $\mathbf{x}_{k \mid T}^{s}$, respectively.

\section{Maximum Likelihood and Maximum a Posteriori Parameter Estimation}

This study utilizes $\mathrm{ML}$ and MAP techniques to estimate the unknown model parameter set $\Theta=$ $\left\{\overline{\mathbf{x}}_{1}, \boldsymbol{\Sigma}_{1}, \mathbf{F}, \mathbf{Q}, \mathbf{R}\right\}$. This parameter set is essential to use the Kalman filter. A database which consists of measured epicardial potentials $\mathbf{X}_{t r}=\left\{\mathbf{x}_{1: T}^{\ell}\right\}_{\ell=1: L}$ and corresponding simulated body surface potentials $\mathbf{Y}_{t r}=\left\{\mathbf{y}_{1: T}^{\ell}\right\}_{\ell=1: L}$ is used by ML- and MAP-based parameter estimation algorithms in this study.

Maximum likelihood estimation: This method estimates the parameter $\Theta$ by maximizing the log likelihood function:

$$
\hat{\mathbf{\Theta}}=\underset{\boldsymbol{\Theta}}{\arg \max } \ln \underbrace{p\left(\mathbf{X}_{t r}, \mathbf{Y}_{t r} \mid \mathbf{\Theta}\right)}_{\mathcal{L}(\boldsymbol{\Theta})} \text {. }
$$

Under the assumption that experiments in the training set are independent and have Markov property [5,6], the log likelihood function can be written as follows:

$$
\begin{aligned}
\ln \mathcal{L}(\boldsymbol{\Theta}) & =\sum_{\ell=1}^{L} \ln p\left(\mathbf{x}_{1}^{\ell} \mid \overline{\mathbf{x}}_{1}, \overline{\mathbf{\Sigma}}_{1}\right)+\sum_{\ell=1}^{L} \sum_{k=1}^{T} \ln p\left(\mathbf{y}_{k}^{\ell} \mid \mathbf{x}_{k}^{\ell}, \mathbf{R}\right) \\
& +\sum_{\ell=1}^{L} \sum_{k=2}^{T} \ln p\left(\mathbf{x}_{k}^{\ell} \mid \mathbf{x}_{k-1}^{\ell}, \mathbf{F}, \mathbf{Q}\right)
\end{aligned}
$$

where

$$
\begin{aligned}
p\left(\mathbf{x}_{1}^{\ell} \mid \overline{\mathbf{x}}_{1}, \mathbf{\Sigma}_{1}\right) & \sim \mathcal{N}\left(\mathbf{x}_{1}^{\ell} ; \overline{\mathbf{x}}_{1}, \mathbf{\Sigma}_{1}\right), \\
p\left(\mathbf{y}_{k}^{\ell} \mid \mathbf{x}_{k}^{\ell}, \mathbf{R}\right) & \sim \mathcal{N}\left(\mathbf{y}_{k}^{\ell} ; \mathbf{A} \mathbf{x}_{k}^{\ell}, \mathbf{R}\right), \\
p\left(\mathbf{x}_{k}^{\ell} \mid \mathbf{x}_{k-1}^{\ell}, \mathbf{F}, \mathbf{Q}\right) & \sim \mathcal{N}\left(\mathbf{x}_{k}^{\ell} ; \mathbf{F} \mathbf{x}_{k-1}^{\ell}, \mathbf{Q}\right) .
\end{aligned}
$$

The parameter $\Theta$ is estimated by taking the derivative of equation 13 with respect to each parameter and equate the derivatives to zero. The resulting estimated parameters are summarized in Table 1.

Table 1. ML based parameter estimation

Estimated parameters:

$$
\begin{aligned}
\hat{\mathbf{x}}_{1}= & \frac{1}{L} \sum_{\ell=1}^{L} \mathbf{x}_{1}^{\ell}, \\
\hat{\mathbf{\Sigma}}_{1}= & \frac{1}{L} \sum_{\ell=1}^{L}\left(\mathbf{x}_{1}^{\ell}-\hat{\overline{\mathbf{x}}}_{1}\right)\left(\mathbf{x}_{1}^{\ell}-\hat{\overline{\mathbf{x}}}_{1}\right)^{T}, \\
\hat{\mathbf{R}}= & \frac{1}{L T} \sum_{\ell=1}^{L} \sum_{k=1}^{T}\left(\mathbf{y}_{k}^{\ell}-\mathbf{A} \mathbf{x}_{k}^{\ell}\right)\left(\mathbf{y}_{k}^{\ell}-\mathbf{A} \mathbf{x}_{k}^{\ell}\right)^{T}, \\
\hat{\mathbf{F}}= & {\left[\sum_{\ell=1}^{L} \sum_{k=2}^{T} \mathbf{x}_{k}^{\ell}\left(\mathbf{x}_{k-1}^{\ell}\right)^{T}\right] } \\
& \cdot\left[\sum_{\ell=1}^{L} \sum_{k=2}^{T} \mathbf{x}_{k-1}^{\ell}\left(\mathbf{x}_{k-1}^{\ell}\right)^{T}\right]^{-1},
\end{aligned}
$$

$\hat{\mathbf{Q}}=\frac{1}{L(T-1)} \sum_{\ell=1}^{L} \sum_{k=2}^{T}\left(\mathbf{x}_{k}^{\ell}-\hat{\mathbf{F}} \mathbf{x}_{k-1}^{\ell}\right)\left(\mathbf{x}_{k}^{\ell}-\hat{\mathbf{F}} \mathbf{x}_{k-1}^{\ell}\right)^{T}$.

Maximum a posteriori estimation: ML estimation is known to suffer from an over-fitting issue due to its nature [7][8]. As a consequence, this may cause major performance degradation. In that case, MAP estimation can be used as an alternative to ML estimation. In MAP estimation, the log posterior pdf is maximized as follows:

$$
\hat{\mathbf{\Theta}}=\underset{\boldsymbol{\Theta}}{\arg \max }\left[\ln p\left(\mathbf{X}_{\mathbf{t r}}, \mathbf{Y}_{\mathbf{t r}} \mid \boldsymbol{\Theta}\right)+\ln \mathbf{p}(\boldsymbol{\Theta})\right](17)
$$

Using Ozbek et al.'s approach [5], we divide the parameter set into two subsets such that $\Theta=\left\{\boldsymbol{\Theta}_{1}, \boldsymbol{\Theta}_{2}\right\}$ where $\boldsymbol{\Theta}_{1}=\left\{\overline{\mathbf{x}}_{1}, \boldsymbol{\Sigma}_{\mathbf{1}}, \mathbf{R}\right\}$ and $\boldsymbol{\Theta}_{2}=\{\mathbf{F}, \mathbf{Q}\}$. $\boldsymbol{\Theta}_{1}$ and $\boldsymbol{\Theta}_{2}$ are assumed to be independent, and $p\left(\boldsymbol{\Theta}_{\mathbf{1}}\right)$ is taken as a non-informative prior. Since the parameter $\Theta_{1}$ is noninformative prior, the estimated values of this parameter set elements are the same as those obtained in the ML estimation. To estimate the parameter $\boldsymbol{\Theta}_{\mathbf{2}}, p(\mathbf{F} \mid \mathbf{Q})$ is assumed to be a zero mean normal distribution, and $p(\mathbf{Q})$ is the inverse Wishart distribution. By plugging in the necessary parameters into equation 17 and taking the derivative of it with respect to $\mathbf{F}$ and $\mathbf{Q}$, the estimated parameters are found and summarized in Table 2. Further mathematical details of ML and MAP estimation techniques can be found in our previous works [3][9]. 
Table 2. MAP based parameter estimation

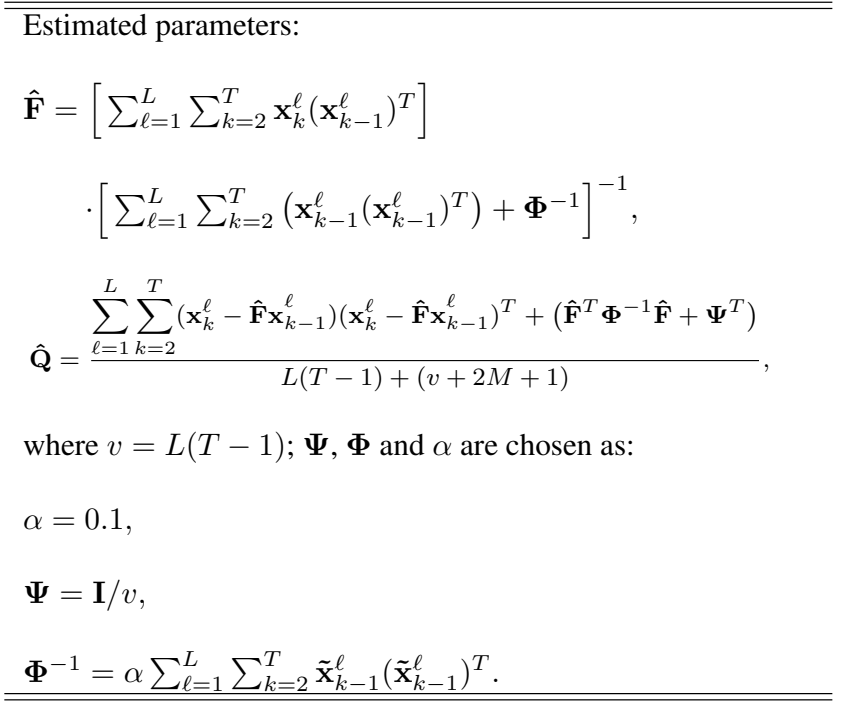

\section{Results and Conclusion}

Three different solution methods are implemented in this study as follows:

- Tikhonov regularization (TIKH): Zero-order Tikhonov regularization is applied to the problem [10].

- Maximum likelihood inference and filtering (MLIF): Kalman filter and smoother is applied to solve the problem. The parameters that are essential to initialize and run the Kalman filter are found by using ML estimation.

- Maximum a posteriori inference and filtering (MAPIF): In this method, again, Kalman filter and smoother is applied. However, this time, the parameters that are essential for the Kalman filter are found by using MAP estimation.

We compose two different training sets consisting of recordings from a different experiment to understand the effect of prior information on the inverse solutions:

- Training Set 1 (Scenario 1): This set consists of epicardial potentials which are in the range of $20 \mathrm{~mm}$. of the test beat's initial stimulation point, at most. The methods that use training set 1 for parameter estimation are labeled as MLIF-1 and MAPIF-1.

- Training Set 2 (Scenario 2): This set consists of epicardial potentials which are in the range of $40 \mathrm{~mm}$. of the test beat's initial stimulation point, at most. The methods that use training set 2 for parameter estimation are labeled as MLIF-2 and MAPIF-2.

In this study, University of Utah's epicardial potential database was used. Body surface potentials were calculated by simulation, i.e., the epicardial potentials were multiplied by the forward transfer matrix, which was found by using boundary element method, and a proper noise (10 $\mathrm{dB}$ or $30 \mathrm{~dB}$ SNR) was added to the result. For all the scenarios, 100 Monte Carlo runs were used to obtain the results. The average of all these 100 simulations was taken in each case.

Quantitative evaluation of electrograms: In Fig. 1 and 2, we present the box plots for the CC when the body surface potentials are simulated at 10 and $30 \mathrm{~dB}$ SNR, respectively. The results show that for both SNR cases, MLIF-2 (mean CC of 0.75 and 0.77, for 10 and $30 \mathrm{~dB}$ SNR cases, respectively) outperforms MLIF-1 (mean CC of 0.73 and 0.75 , for 10 and $30 \mathrm{~dB}$ SNR cases, respectively). As per these results, we can conclude that using a more general training set with pacing sites spreading to larger area yields better results in the ML-based method. On the other hand, MAPIF-1 and MAPIF-2 show almost the same performances, MAPIF-1 gives mean CC of 0.77 and 0.81 ; MAPIF-2 gives mean $\mathrm{CC}$ of 0.76 and 0.81 for 10 and $30 \mathrm{~dB}$ SNR cases, respectively. Tikhonov regularization is the most vulnerable method to the measurement noise and its mean CC value drops by $16 \%$ as SNR decreases from 30 to $10 \mathrm{~dB}$.

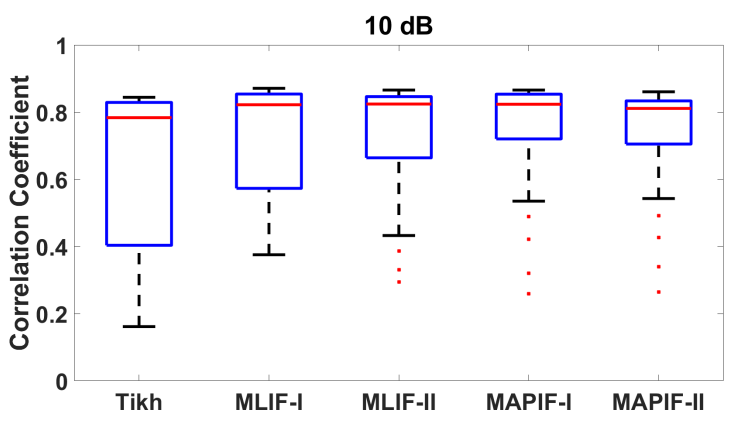

Figure 1. Box plots for the $\mathrm{CC}$ values; BSPs are simulated at $10 \mathrm{~dB} \mathrm{SNR}$

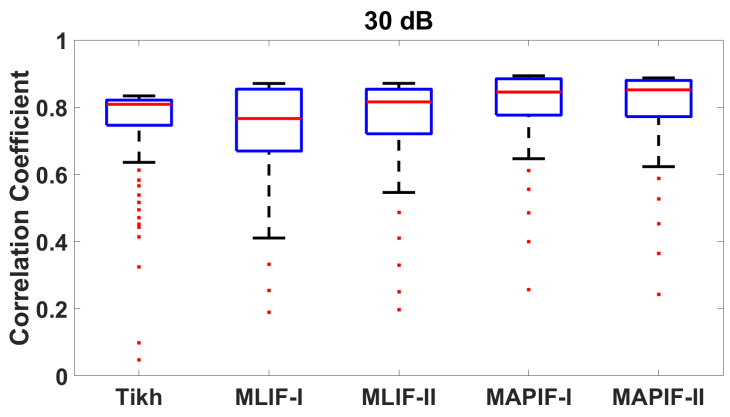

Figure 2. Box plots for the $\mathrm{CC}$ values; BSPs are simulated at $30 \mathrm{~dB}$ SNR

Evaluation of activation times and localization error: In this section, the performances of the proposed methods are investigated in terms of activation times and localization errors. Tables 3 and 4 show the $\mathrm{CC}$ values of the activation times and localization errors, respectively. As seen in Table 3, in general, the methods that use training 
set 1 give higher $\mathrm{CC}$ values in terms of activation times. MAPIF-1 is the most robust method to the measurement noise and its $\mathrm{CC}$ value drops approximately by $3 \%$. In terms of localization errors, Tikhonov regularization is the most fragile method to the measurement noise and its localization error increases from $5.06 \mathrm{~mm}$ to $38.58 \mathrm{~mm}$ when the measurement noise is changed $30 \mathrm{~dB}$ to $10 \mathrm{~dB}$ SNR. In $10 \mathrm{~dB}$ SNR case, MLIF, which provides a localization error of $4.75 \mathrm{~mm}$ at most, outperforms MAPIF, which provides $9.48 \mathrm{~mm}$ at most. On the other hand, in $30 \mathrm{~dB}$ SNR case, MAPIF-1 outperforms the other methods and finds the origin of the beat within a range of $4.75 \mathrm{~mm}$.

Table 3. CC values for ATs for all methods; BSPs are simulated at 30 and $10 \mathrm{~dB}$ SNR

\begin{tabular}{lcc}
\hline \hline Method & $10 \mathrm{~dB}$ & $30 \mathrm{~dB}$ \\
\hline Tikhonov & 0.78 & 0.81 \\
MLIF-1 & 0.82 & 0.93 \\
MLIF-2 & 0.77 & 0.82 \\
MAPIF-1 & 0.89 & 0.92 \\
MAPIF-2 & 0.75 & 0.85 \\
\hline \hline
\end{tabular}

Table 4. Localization errors (in $\mathrm{mm}$ ) for all methods; BSPs are simulated at 30 and $10 \mathrm{~dB}$ SNR

\begin{tabular}{lcc}
\hline \hline Method & $10 \mathrm{~dB}$ & $30 \mathrm{~dB}$ \\
\hline Tikhonov & 38.58 & 5.06 \\
MLIF-1 & 4.75 & 5.89 \\
MLIF-2 & 0 & 5.89 \\
MAPIF-1 & 9.48 & 4.75 \\
MAPIF-2 & 9.48 & 14.47 \\
\hline \hline
\end{tabular}

\section{Discussions and Future Works}

In this study, the prior information effects on the solution of inverse electrocardiography was studied. Since ML-based methods have over-fitting issue due to their nature, MAPIF yielded more accurate solutions as compared to MLIF. Furthermore, we observed that using a training set which covers a wider region in terms of the pacing locations, i.e training set 2 , slightly improved the electrogram reconstructions. Increasing the diversity of epicardial distributions in the training process made a positive impact on the results and provided more regularized and smoother solutions. On the other hand, training sets which were composed by choosing the beats that have pacing sites close to the test beat's pacing location, i.e training set 1 , were more successful in finding activation times and yielded less localization errors. This was an expected result because the closer beats have more similar distributions to the test beat in the early times, increasing the accuracy of the reconstructions early in the activation.

\section{Acknowledgments}

This work was supported by The Scientific and Technological Research Council of Turkey, grant number 118E244. The authors would like to thank Dr. Robert S. MacLeod and his colleagues from University of Utah, Nora Eccles Harrison Cardiovascular Research and Training Institute for the data used in this study.

\section{References}

[1] Serinagaoglu, Y., Brooks, D. H., MacLeod, R. S. (2006). Improved performance of Bayesian solutions for inverse electrocardiography using multiple information sources.IEEE Transactions on Biomedical Engineering, 53(10), 2024-2034.

[2] Suarez-Gutierrez, V. et al.(2017). Including a priori knowledge in the solution of the inverse problem during atrial fibrillation. In 2017 Computing in Cardiology (CinC) (pp. 1-4).

[3] Erenler, T., Serinagaoglu Dogrusoz, Y. (2019). ML and MAP estimation of parameters for the Kalman filter and smoother applied to electrocardiographic imaging. Medical \& Biological Engineering \& Computing.

[4] Rauch, H. E., Striebel, C. T., Tung, F. (1965). Maximum likelihood estimates of linear dynamic systems. AIAA Journal, 3(8), 1445-1450.

[5] Yücel Özbek, I., Demirekler, M. (2010). ML vs. MAP parameter estimation of linear dynamic systems for acousticto-articulatory inversion: A comparative study. European Signal Processing Conference, 805-809.

[6] Ghahramani, Z., Hinton, G. E. (1996). Parameter Estimation for Linear Dynamical Systems. Technical Report, 6(CRG-TR-96-2), 1-6.

[7] Bishop, C. M. (2006). Pattern Recognition and Machine Learning (Information Science and Statistics). Berlin, Heidelberg: Springer-Verlag.

[8] Erenler, T., Serinagaoglu Dogrusoz, Y. (2018). A Learning Based Statistical Approach for Combining Multiple Measurements in Electrocardiographic Imaging. In 2018 Computing in Cardiology Conference (CinC) (Vol. 45, pp. 1-4).

[9] Erenler, T. (2018). Comparison of ML and MAP parameter estimation techniques for the solution of inverse electrocardiography problem. Middle East Technical University.

[10] Tikhonov, A. N., Arsenin, V. I. A. (1977). Solutions of IllPosed Problems. Halsted. New York.

Adress for correspondence:

Assoc. Prof. Dr. Yeşim Serinağaoğlu Doğrusöz, Electrical and Electronics Engineering Department, Middle East Technical University, yserin@metu.edu.tr 